Pacific Journal of Mathematics

OPERATOR CALCULUS 


\section{OPERATOR CALCULUS}

\section{Philip FeInsilver}

To an analytic function $L(z)$ we associate the differential operator $L(D), D$ denoting differentiation with respect to a real variable $x$. We interpret $L$ as the generator of a process with independent increments having exponential martingale $m(x(t), t)=\exp (z x(t)-t L(z))$. Observing that $m(x$, $-t)=e^{z C} 1$ where $C=e^{t L} x e^{-t L}$, we study the operator calculus for $C$ and an associated generalization of the operator $x D, A=C D$. We find what functions $f$ have the property that $u_{n}=C^{n} f$ satisfy the evolution equation $u_{t}=L u$ and the eigenvalue equations $A u_{n}=n u_{n}$, thus generalizing the powers $x^{n}$. We consider processes on $R^{N}$ as well as $R^{1}$ and discuss various examples and extensions of the theory.

In the case that $L$ generates a Markov semigroup, we have transparent probabilistic interpretations. In case $L$ may not generate a probability semigroup, the general theory gives some insight into what properties any associated "processes with independent increments" should have. That is, the purpose is to elucidate the Markov case but in such a way that hopefully will lead to practicable definitions and will present useful ideas for defining more general processes-involving, say, signed and/or singular measures.

II. Probabilistic basis. Let $p_{t}(x)$ be the transition kernel for a process $\rho(t)$ with stationary independent increments. That is,

$$
\int_{A} p_{t}(x)=\operatorname{Prob}(\rho(t) \in A \mid \rho(0)=0) .
$$

The Lévy-Khinchine formula says that, generally:

$$
\int_{R} e^{i \xi x} p_{t}(x)=e^{t L(i \hat{\xi})}
$$

where $L(i \xi)=a i \xi-\sigma^{2} \xi^{2} / 2+\int_{R-\{0\}} e^{i \xi u}-1-i \xi \eta(u) \cdot M(d u)$ with

$$
\eta(u)=u(|u| \leqq 1)+\operatorname{sgn} u(|u| \geqq 1) \text { and } \int \frac{u^{2}}{1+u^{2}} M(d u)<\infty \text {. }
$$

Denoting $d / d x$ by $D$, this states that $L(D)$ is the generator of the process $\rho(t)$. It follows that

$$
m(t)=e^{\alpha \rho(t)-t L(z)} \text { is a martingale }
$$


for every $z \in C, \operatorname{Rez}=0$. And this is clearly equivalent to the condition that $L$ generates $\rho$. We define $h_{k}(x, t)$ by

$$
e(z)=e^{z x+t L(z)}=\sum_{0}^{\infty} \frac{z^{k}}{k !} h_{k}(x, t) .
$$

Observe that $\partial e / \partial z=\left(x+t L^{\prime}(z)\right) e(z)$ which is the basis for $\S I V$. Note that this expansion is good only in case the distribution of $\rho(t)$ has all moments finite. In fact, define moment functions

$$
h_{k}(x, t)=\int_{R}(x+y)^{k} p_{t}(y)=e^{t L(D)} x^{k} .
$$

And check that,

$$
\sum_{0}^{\infty} \frac{z^{k}}{k !} h_{k}(x, t)=e^{z x} \int_{R} e^{z y} p_{t}(y)=e^{z x+t L(z)} .
$$

Thus if $p_{t}(x)$ has only moments up to order $\alpha$, then define $h_{k}$ for $0 \leqq k<\alpha$, say. Also, $k$ need not be restricted to integer values, but generally $k \in \boldsymbol{R}, k \geqq 0$. We set $H_{k}(x, t)=h_{k}(x,-t)$.

Note that since for $t>0, s<t$,

$$
E\left(e^{z \rho(t)-t L(z)} \mid 0 \leqq \rho(u) \leqq s\right)=e^{z \rho(s)-s L(z)}
$$

then $E\left(H_{k}(\rho(t), t) \mid 0 \leqq \rho(u) \leqq s\right)=H_{k}(\rho(s), s)$. That is, $H_{k}(\rho(t), t)$ is a martingale for every $k$. From the above remarks it follows:

(1) $h_{k}(x, t)=E(x+\rho(t))^{k}, t \geqq 0$.

(2) $E h_{k}(x+\rho(t), s)=h_{k}(x, t+s), t, s \geqq 0$.

(3) $E h_{k}(x+\rho(t),-t)=x^{k}, t \geqq 0$.

In the following we develop an operator calculus associated with these $h_{k}$ and study various properties and extensions based upon the above preliminaries.

\section{Notations.}

(1) $d / d x$ will be denoted by $D$.

(2) $L(D)$ will be "any" function of $D$ that is thought of as the generator of a process with independent increments. $L$ is assumed to have constant coefficients (independent of $x$ and $t$ ); and generally $L(0)=0$.

(3) $E X$ or $\langle X\rangle$ will be used to denote expected value.

(4) $t$, the "time", is independent of all $x$ and $D$ variables. Generally $t \geqq 0$.

Other notations are standard or will be explained as they arise. The functional calculus for $D$ will be implemented by Fourier transform as needed to facilitate and clarify computations. 
IV. Algebraic structure. Assume given an operator $C$ such that

$$
[D, C]=D C-C D=1 .
$$

For example, $C=x$ as a multiplication operator. By induction it follows readily that

$$
\left[D^{r}, C\right]=r D^{r-1}
$$

and

$$
\left[D, C^{r}\right]=r C^{r-1}, \quad r>0 .
$$

Thus for any polynomial or analytic function $\phi(D)$,

$$
[\phi(D), C]=\phi^{\prime}(D),
$$

$\phi^{\prime}$ denoting the derivative of $\dot{\phi}$. So, even for nondifferentiable $\phi$, denote $[\phi(D), x]$ by $\phi^{\prime}$.

Proposition. Set $C=x+t[\phi(D), x] . \quad$ Then $[D, C]=1$.

Proof.

$$
\begin{aligned}
D C & =D x+t D \phi(D) x-t D x \phi(D) \\
& =x D+1+t \phi(D) D x-t x \phi(D) D-t \phi(D) \\
& =x D+1+t \phi(D) x D+t \phi(D)-t x \phi(D) D-t \phi(D) \\
& =(x+t[\phi(D), x]) D+1 .
\end{aligned}
$$

Since $[D, x]=1$, this checks that $\left[D, \phi^{\prime}\right]=\left[\phi, \phi^{\prime}\right]=0$. Denote by $A$ the operator $C D$. Then the following hold (by induction).

$$
\begin{aligned}
& {\left[A, C^{k}\right]=k C^{k}} \\
& {\left[A^{k}, C\right]=C\left((A+1)^{k}-A^{k}\right) .}
\end{aligned}
$$

Similarly,

$$
\left[D^{k}, A\right]=k D^{k}
$$

and

$$
\left[D, A^{k}\right]=\left((A+1)^{k}-A^{k}\right) D .
$$

Given a function $\phi(D)$, such that $\phi(D) 1=0$, set $C=x+t \phi^{\prime}$. Assume $A 1=0$. Define $h_{k}(x, t)=C^{k} 1$. Then the following properties hold:

(1) $C h_{k}=h_{k+1}$.

(2) $D h_{k}=k h_{k-1}$.

(3) $A h_{k}=C D h_{k}=k h_{k}$. 
(4) $\partial h_{k} / \partial t=\phi(D) h_{k}$.

(5) $h_{k}(x, 0)=x^{k}$.

The generating function $e(z)=\sum_{0}^{\infty}\left(z^{k} / k !\right) h_{k}$ has these properties:

(6) $\partial e / \partial z=C e, e(0)=1$.

(7) $C^{k} e(z)=e^{z C} h_{k}$.

The above are easy to check using the commutation relations noted previously. It is worthwhile to check $\# 4$ explicitly:

For $k=0, h_{k} \equiv 1$ and $\partial h_{k} / \partial t=0=\phi(D) 1$.

For $k=n+1, h_{k}=C h_{n}$ and

$$
\begin{aligned}
\frac{\partial h_{k}}{\partial t} & =\frac{\partial C}{\partial t} h_{n}+C \frac{\partial h_{n}}{\partial t} \\
& =\phi^{\prime} h_{n}+C \phi h_{n} \\
& =(\phi C-C \phi) h_{n}+C \phi h_{n} \\
& =\phi C h_{n}=\phi h_{k},
\end{aligned}
$$

since

$$
\dot{\phi}^{\prime}=[\phi, x]=[\phi, C] .
$$

The condition $\phi 1=0$ comes only in proving $\# 4$; the condition $A 1=0$ for $\sharp 2$. $\sharp 3$ follows from $\sharp 1$ and $\sharp 2$. These $h_{k}$ thus generalize the powers $x^{k}$.

REMARK. In case $\phi$ is analytic, $\phi(D)=\sum_{0}^{\infty} a_{p} D_{p} / p$ !, the $h_{k}$ are the determinants of a family of matrices of dimension $k$. This is seen as follows. Set $\bar{C} \equiv x-t \phi^{\prime} . H_{k}(x, t) \equiv h_{k}(x,-t)=\bar{C}^{k} 1$. Then,

$$
\begin{aligned}
x H_{k} & =t \phi^{\prime} H_{k}+H_{k+1}=t \sum_{1}^{\infty} a_{p} \frac{D^{p-1}}{(p-1) !} H_{k}+H_{k+1} \\
& =t \sum_{1}^{\infty} a_{p} \frac{k !}{(p-1) !(k-p+1) !} H_{k-p+1}+H_{k+1} \\
& =t \sum_{0}^{k} a_{p+1}\left[\begin{array}{l}
k \\
p
\end{array}\right] H_{k-p}+H_{k+1} .
\end{aligned}
$$

Thus, $H_{k}=\operatorname{det}\left(x-A_{k}\right)$ where the matrix $A_{k}$ has entries:

$$
A_{k, r s}=\begin{array}{cc}
1 & s=r+1 \\
0 & s>r+1 \\
t\left[\begin{array}{l}
r-1 \\
s-1
\end{array}\right] a_{r-s+1} s \leqq r+1 .
\end{array}
$$

In the probabilistic case, $\phi$ is chosen to be the generator $L$. Then the moment polynomials $h_{k}$ can be computed as $C^{k} 1$. The relations $\left\langle h_{k}(\rho(t),-t)\right\rangle=0$ yield the moments $\mu_{k}=\int y^{k} P_{t}(y)$ and, conversely, 
the original definition yields the $h_{k}$ in terms of the $\mu_{k}, h_{k}=$ $\Sigma\left[\begin{array}{l}k \\ r\end{array}\right] \mu_{r} x^{k-r}$.

EXAMPLES.

1. $L=D . C=x+t . A=(x+t) D . h_{k}=(x+t)^{k}$.

2. Brownian motion. $L=(1 / 2) D^{2} . C=x+t L^{\prime}=x+t D . A=$ $x D+t D^{2}$.

$$
h_{k}=\int_{R}(x+y)^{k} \frac{e^{-y^{2 / 2 t}}}{\sqrt{2 \pi t}} d y
$$

and $H_{k}$ are the Hermite polynomials.

3. Poisson process. $L=e^{D}-1$. $C=x+t e^{D}$. The first few polynomials are:

$$
\begin{aligned}
& h_{0}=1 . h_{1}=x+t . \\
& h_{2}=x^{2}+(2 x+1) t+t^{2} . \\
& h_{3}=x^{3}+\left(3 x^{2}+3 x+1\right) t+(3 x+3) t^{2}+t^{3} .
\end{aligned}
$$

4. Singular case. $L=\log D$. Then $C=x+t D^{-1} \cdot A=x D+t$. The equations $A h_{k}=k h_{k}$ become:

yielding

$$
x h^{\prime}+t h=k h
$$

$$
h_{k}=a x^{k-t} .
$$

Choosing $h_{0}=x^{-t}$ gives:

$$
h_{k}=\frac{k ! x^{k-t}}{(1-t)(2-t) \cdots(k-t)}=\frac{(-t) ! k ! x^{k-t}}{(k-t) !}
$$

defining $q ! \equiv \Gamma(q+1)$.

V. The operator C. As in $\S I V$, given a generator $L(D)$, define $C=x+t L^{\prime}$. For general functions $L(D), L(0)=0$, set

$$
p_{t}(x)=\frac{1}{2 \pi} \int_{R} e^{-i \xi x} e^{t L(i \xi)} \text { whenever this }
$$

may be defined; e.g., if necessary, as a distribution.

Proposition. The solutions to $C f=\lambda f$ are of the form

$$
f=k(t) p_{t}(\lambda-x) \text {. }
$$

Proof.

$x f+t L^{\prime} f=\lambda f$ becomes by Fourier transformation

$$
i \frac{d}{d \xi} \hat{f}+t L^{\prime}(i \xi) \hat{f}=\lambda \hat{f}
$$


which has the solution $\hat{f}=k(t) e^{-i \lambda \xi} e^{t \int_{0}^{\xi} L^{\prime}(i s) i d s}=k(t) e^{-i \lambda \xi} e^{t L(i \xi)}$.

REMARK. Recall from IV that as long as $A h_{0}=0, A h_{k}=k h_{k}$. This indicates that there is a $2 n d$ series, besides $C^{k} 1$, formed as follows. Take $h_{0}=D^{-1} p_{t}(-x), D^{-1}$ denoting indefinite integral. Then by the above proposition with $\lambda=0, A h_{0}=C D h_{\mathrm{r}}=0$. Now define $h_{k}=C^{k} h_{0}$. Then $h_{k-1}$ is actually $k$ ! times the $k$ th indefinite integral of $p_{t}(-x)$ and $A h_{k}=k h_{k}$.

Now proceed to study in more detail the operator $C$ acting on functions in general.

Definition. A function $f(x, t)$ is harmonic by definition when

$$
\frac{\partial f}{\partial t}=L f \text {. }
$$

Then it will be seen that:

THEOREM. If $f$ is harmonic, then $u=e^{z c} f$ is harmonic. Defining $f_{k}=C^{k} f$, then

CoROLlaRY. If $f$ is harmonic, then $f_{k}$ is harmonic for all $k \geqq 0$. In fact, $e^{z C} f$ harḿonic means

$$
\frac{\partial}{\partial t} e^{z C} f=L e^{z C} f \text { and to conclude that } f_{k} \text { is }
$$

harmonic note that differentiating with respect to $z$ commutes with both $\partial / \partial t$ and $L$.

First compute $e^{z C} f$.

Lemma 1. Set $\Lambda(D)=L(D+z)-L(D)$. Then

$$
e^{z C} f=e^{z x} e^{t \Lambda} f \text {. }
$$

Proof. Let $\psi=L^{\prime}$. For $u=e^{z C} f$,

$$
\frac{\partial u}{\partial z}=C u=x u+t \psi u \text {. }
$$

Apply Fourier transformation to yield, for $v=\widehat{u}$ :

$$
\frac{\partial v}{\partial z}=i \frac{\partial v}{\partial \xi}+t \psi(i \xi) v
$$

which has for solution

$$
v=\widehat{f}(\xi+i z) e^{t} \int_{0}^{z} \psi(i \xi-s) d s
$$


as is readily seen, say, by applying Feynman-Kac for the deterministic process $\rho(z)=i z$. Thus,

$$
\begin{aligned}
& u=\int e^{i \xi x} \hat{f}(\xi+i z) e^{t} \int_{0}^{z} \psi(i \xi-s) d s
\end{aligned}
$$

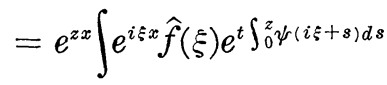

$$
\begin{aligned}
& =e^{z x} e^{t \int_{0}^{z} \psi(D+s) d s} f .
\end{aligned}
$$

Since $\psi=L^{\prime}, \int_{0}^{z} \psi(D+s) d s=L(D+z)-L(D)$.

The theorem will require the aid of the following.

LEMMA 2. For any $\dot{\phi}(D)$,

$$
\phi(D) e^{z x} f=e^{z x} \phi(D+z) f .
$$

Proof. Utilizing Fourier transformation for the left-hand side,

$$
\begin{aligned}
\dot{\phi}(D) e^{z x} f & =\int e^{i \xi x} \phi(i \xi) \int e^{-i \xi a} e^{z a} f(a) \\
& =\int e^{i \xi x} \phi(i \xi) \hat{f}(\xi+i z) \\
& =\int e^{i \xi x} e^{z x} \phi(i \xi+z) \hat{f}(\xi) \\
& =e^{z x} \phi(D+z) f
\end{aligned}
$$

Finally the proof of the theorem:

Let $\partial f / \partial t=L f$. Then, for $u=e^{z C} f$,

$$
\begin{aligned}
\frac{\partial u}{\partial t} & =\frac{\partial}{\partial t}\left(e^{z x} e^{t} f\right)=e^{z x} e^{t} \Lambda f+e^{z x} e^{t \cdot s} L f \\
& =e^{z x} e^{t}(L(D+z)-L(D)+L(D)) f=e^{z x} e^{t} L(D+z) f \\
& =L u \text { by Lemma } 2 .
\end{aligned}
$$

Remarks. 1. Particularly, when $L 1=0, e(z)=e^{z C} 1$ is harmonic. In fact,

$$
\begin{aligned}
e^{z C} 1 & =e^{z x} e^{t(L(D+z)-L(D))} 1=e^{z x} e^{t L(D+z)} 1 \\
& =e^{t L(D)} e^{z x} 1, \text { by Lemma } 2, \\
& =e^{t L(z)} e^{z x} \text { which is the required result. }
\end{aligned}
$$

2. Note that by Lemma 2, generally,

$$
\begin{aligned}
e^{z C} f & =e^{z x} e^{t L(D+z)} e^{-t L} f \\
& =e^{t L} e^{z x} e^{-t L} f
\end{aligned}
$$


Differentiating with respect to $t$ yields an alternative proof of the theorem. See $\S I X$.

3. In the probabilistic case, the theorem implies that if $f$ is harmonic, $u=e^{z C} f$, then $f_{k}(\rho(t),-t)$ and $u(\rho(t),-t)$ are martingales.

EXAMPLES.

1. Brownian motion.

$$
\Lambda=L(D+z)-L(D)=\frac{1}{2} z^{2}+z D .
$$

So

$$
e^{z C} f=e^{z x} e^{z^{2} t / 2} f(x+z t) .
$$

2. Poisson process.

$$
\Lambda=e^{D}\left(e^{z}-1\right)
$$

So

$$
e^{z C} f=e^{z x} \sum_{0}^{\infty} \frac{t^{n}\left(e^{z}-1\right)^{n}}{n !} f(x+n) .
$$

3. Singular process.

$$
\begin{aligned}
& \Lambda=\log (D+z)-\log D=\log \left(1+z D^{-1}\right) . \\
& e^{z C} f=e^{z x}\left(1+z D^{-1}\right)^{t} f \\
&=e^{z x} \sum\left[\begin{array}{l}
t \\
k
\end{array}\right] z^{k} D^{-k} f .
\end{aligned}
$$

And

$$
f_{k}=\sum_{r}\left[\begin{array}{l}
t \\
r
\end{array}\right] \frac{k ! x^{k-r}}{(k-r) !} D^{-r} f
$$

4. $L(D)=D^{3} / 6 . \quad \Lambda=(1 / 2) z D^{2}+(1 / 2) z^{2} D+(1 / 6) z^{3}$.

$$
e^{z C} f=e^{z x} e^{z^{3 t} / 6} \int f\left(x+\frac{z^{2} t}{2}+\sqrt{z t y}\right) \frac{e^{-y^{2} / 2}}{\sqrt{2 \pi}} d y .
$$

VI. The operator A. Given $C$, define $A=C D$.

This section continues the previous one to analyze the eigenvalue equations $A f_{k}=k f_{k}$.

Proposition. $A f=0$ if and only if $A f_{k}=k f_{k}$, for $k \geqq 0$.

Proof. Set $u=e^{z c} f$. 


$$
\begin{aligned}
A u & =C D e^{z x} e^{t A} f \\
& =C\left(z u+e^{z C} D f\right) \\
& =z C u+e^{z C} A f \\
& =z C u \\
& =z \frac{\partial}{\partial z} u .
\end{aligned}
$$

Thus, $z(\partial / \partial z) z^{k}=k z^{k}$ implies $A C^{k} f=k C^{k} f$. This follows also from the commutation relation $\left[A, C^{k}\right]=k C^{k}$.

To see how $A$ acts on functions in general, solve the equation

$$
\frac{\partial u}{\partial \alpha}=A u
$$

thus computing $e^{\alpha A} f$.

Proposition.

$$
e^{\alpha A} f=e^{\alpha x D} e^{t G} f
$$

where

$$
G=L\left(D e^{\alpha}\right)-L(D)
$$

This is analogous to the result for $C$, replacing translation by $z$ with multiplication, exponential translation.

Proof. Apply Fourier transformation to

$$
\frac{\partial u}{\partial \alpha}=x \frac{\partial u}{\partial x}+t \psi \frac{\partial u}{\partial x}
$$

yielding, for $v=\widehat{u}$,

$$
\begin{aligned}
\frac{\partial v}{\partial \alpha} & =i \frac{\partial}{\partial \xi}(i \xi v)+t \psi(i \xi) i \xi v \\
& =-\xi \frac{\partial v}{\partial \xi}+(t \psi(i \xi) i \xi-1) v .
\end{aligned}
$$

The solution is seen to be

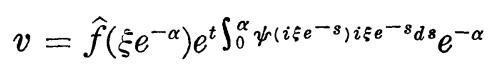

by applying, say, Feynman-Kac for the deterministic process $\rho(\alpha)=$ $\xi e^{-\alpha}$. Thus, 


$$
\begin{aligned}
u & =\int e^{i \xi x} \hat{f}\left(\xi e^{-\alpha}\right) e^{t \int_{0}^{\alpha} \psi\left(i \xi e^{-s}\right) i \xi e^{-s} d s} e^{-\alpha} d \xi \\
& =\int e^{i \xi e^{\alpha} x} \hat{f}(\xi) e^{t \int_{0}^{\alpha} \psi\left(i \xi e^{\alpha-s}\right) i \xi e^{\alpha-s} d s} \\
& =e^{t \int_{0}^{\alpha} \psi\left(D e^{s}\right) D e^{s} d s} f\left(x e^{\alpha}\right) .
\end{aligned}
$$

Setting $t=0$ yields $e^{\alpha x D} f(x)=f\left(x e^{\alpha}\right)$.

Substituting $\psi=L^{\prime}$,

$$
\int_{0}^{\alpha} L^{\prime}\left(D e^{s}\right) D e^{s} d s=L\left(D e^{\alpha}\right)-L(D) .
$$

Combining these two propositions gives

THEOREM. $A f=0$ if and only if

$$
e^{\alpha x D} e^{t G} f_{k}=e^{\alpha k} f_{k} \quad \text { for all } k \geqq 0,
$$

where $G=L\left(D e^{\alpha}\right)-L(D)$.

EXAMPLES.

1. $L=D . A=(x+t) D$. And

$$
e^{\alpha .4} f=e^{t\left(e^{\alpha}-1\right) D} f\left(x e^{\alpha}\right)=f\left(x e^{\alpha}+t e^{\alpha}-t\right) .
$$

Eigenfunctions are $(x+t)^{k}$.

2. Brownian motion. $A=x D+t D^{2}$.

$$
G=\frac{1}{2} D^{2}\left(e^{2 \alpha}-1\right) \text {. }
$$

And

$$
e^{\alpha A} f=\int f\left(x e^{\alpha}+y \sqrt{\left.t\left(e^{2 \alpha}-1\right)\right)} \frac{e^{-y^{2 / 2}}}{\sqrt{2 \pi}} d y\right.
$$

Eigenfunctions are $h_{k}(x, t)$ where $h_{k}(x,-t)$ are Hermite polynomials. This is essentially the Ornstein-Uhlenbeck process.

3. Poission process. $A=x D+t e^{D} D$.

$$
G=e^{D e^{\alpha}}-e^{D} \text {. }
$$

And

$$
e^{\alpha A} f=\sum_{0}^{\infty} \frac{t^{k}}{k !} \sum_{p}\left[\begin{array}{l}
k \\
p
\end{array}\right](-1)^{k-p} f\left(p e^{\alpha}+k-p+x e^{\alpha}\right) .
$$

4. Singular process. $A=x D+t$.

$$
G=\log D e^{\alpha}-\log D=\alpha .
$$


And

$$
e^{\alpha A} f=e^{\alpha t} f\left(x e^{\alpha}\right) .
$$

Eigenfunctions are $x^{k-t}$, as seen previously.

5. Cauchy process. $L=-|D|$.

$$
G=-|D|\left(e^{\alpha}-1\right) \text {. }
$$

And

$$
e^{\alpha A} f=\frac{1}{\pi} \int f\left(x e^{\alpha}+t\left(e^{\alpha}-1\right) y\right) \frac{d y}{1+y^{2}} .
$$

For example, set

$$
h(x)=\frac{1}{\pi} \int \sqrt{x+y t} \frac{d y}{1+y^{2}}
$$

Then

$$
\frac{1}{\pi} \int h\left(x e^{\alpha}+t\left(e^{\alpha}-1\right) y\right) \frac{d y}{1+y^{2}}=e^{\alpha / 2} h(x), \text { for all } \alpha \geqq 0 .
$$

VII. Multidimensional theory. In the case where $x \in \boldsymbol{R}^{N}$ and $D=\left(D_{1}, D_{2}, \cdots, D_{N}\right)$ the exponential martingale is

$$
e^{z \cdot \rho-t L(z)} .
$$

The natural extension of the one-dimensional operator $C$ is the vector operator

$$
C=\left(C_{1}, \cdots, C_{N}\right)
$$

with components

$$
C_{j}=x_{j}+t\left[L, x_{j}\right]=x_{j}+t \frac{\partial L}{\partial D_{j}} \equiv x_{j}+t L_{j} .
$$

The generating function

$$
e^{z \cdot x+t L(Z)}=\sum_{k} \frac{z^{k}}{k !} h_{k}(x, t)
$$

where the usual indexing notations, e.g., $z^{k}=z_{1}^{k_{1}} \cdots z_{N}^{k_{N}}$, are employed, is again represented as $e^{z \cdot C} 1$. The corresponding basic theorem of $\S \mathrm{V}$ will be the main topic of this section.

Theorem. Given $L(D)=L\left(D_{1}, \cdots, D_{N}\right)$. For $C$ as defined above, $f$ harmonic implies $e^{z \cdot c} f$ is harmonic.

The proof will follow from the explicit form of $u=e^{z \cdot c} f$. 
Proposition. The operators $C_{j}$ commute.

Proof.

Let $C_{1}=x+t L_{x}$ and $C_{2}=y+t L_{y}$ be two $C_{j}$ 's. Then

$$
\begin{aligned}
C_{1} C_{2} & =\left(x+t L_{x}\right)\left(y+t L_{y}\right) \\
& =x y+t x L_{y}+t L_{x} y+t L_{x} L_{y} \\
& =x y+t x L_{y}+t y L_{x}+t \frac{\partial L_{x}}{\partial D_{y}}+t L_{x} L_{y} \\
& =x y+t x L_{y}+t y L_{x}+t L_{x y}+t L_{x} L_{y}
\end{aligned}
$$

which is symmetric in $x$ and $y$ since $L_{x y}=L_{y x}$.

By the proposition,

$$
e^{z \cdot C} f=\prod_{j} e^{z_{j} j_{j} f} \text { (independently of the order of the product) }
$$

and by the results of $\S \mathrm{V}$,

$$
e^{z \cdot C} f=e^{z_{1} x_{1}} e^{t}{ }_{1}^{z_{2} x_{2}} e^{t}{ }^{2} \cdots e^{z_{N}{ }^{x} N} e^{t \Lambda_{N}} f
$$

where

$$
\Lambda_{j}=L\left(D_{1}, \cdots, D_{j}+z_{j}, \cdots, D_{N}\right)-L(D) .
$$

Similarly, the operators $A_{j}=x_{j} D_{j}+t L_{j} D_{j}$ commute and

$$
e^{\alpha \cdot A} f=e^{\alpha_{1} x_{1} D_{1}} e^{t G_{1}} \cdots e^{\alpha_{N} x_{N} D_{N}} e^{t G_{N}} f
$$

where

$$
G_{j}=L\left(D_{1}, \cdots, D_{j} e^{\alpha j}, \cdots, D_{N}\right)-L(D)
$$

Proof of the harmonicity theorem. For $u=e^{z \cdot c} f$,

$$
\begin{gathered}
\frac{\partial u}{\partial t}=\sum_{j} e^{z_{1} x_{1}} e^{t \Lambda_{1}} \cdots e^{z_{j} x_{j}} e^{t_{\Lambda_{j}}} \Lambda_{j} e^{z_{j+1} x_{j+1}} e^{t \Lambda_{j+1}} \cdots e^{z_{N} x_{N}} e^{t_{N}} f \\
+\prod_{j} e^{z_{j} x_{j}} e^{t^{\prime} j} \frac{\partial f}{\partial t} .
\end{gathered}
$$

While, using Lemma 2 of $\S \mathrm{V}$ sequentially (i.e., by induction),

$$
\begin{aligned}
L u= & L e^{z_{1} x_{1}} e^{t \Lambda_{1}} \cdots e^{z_{N} x_{N}} e^{t \Lambda_{N} f} \\
= & e^{z_{1} x_{1}} e^{t \Lambda_{1}}\left(\Lambda_{1}+L\right) e^{z_{2} x_{2}} \cdots e^{t \Lambda_{N}} f \\
= & e^{z_{1} x_{1}} e^{t \Lambda_{1}} \Lambda_{1} e^{z_{2} x_{2}} \cdots f+e^{z_{1} x_{1}} e^{t \Lambda_{1}} L e^{z_{2} x_{2}} e^{t \Lambda_{2}} \cdots e^{t \Lambda_{N} f} \\
= & \sum_{j} e^{z_{1} x_{1}} e^{t{ }_{1}} \cdots e^{z_{j} x_{j}} e^{t \Lambda_{j}} \Lambda_{j} \cdots e^{z_{N} x_{N}} e^{t \Lambda_{N}} f \\
& +\prod_{j} e^{z_{j} x_{j}} e^{t \Lambda_{j}} L f \\
= & \frac{\partial u}{\partial t} .
\end{aligned}
$$


REMARKS 1. Using Remark 2 of $\S \mathrm{V}$, it is clear that

$$
e^{z \cdot c} f=\prod_{j} e^{t L} e^{z_{j} x_{j}} e^{-t L} f=e^{t L} e^{z^{* x}} e^{-t L} f .
$$

2. As before, check that, for $L 1=0$, the exponential generating function satisfies

$$
e^{z \cdot x+t L(z)}=e^{z \cdot C} 1 .
$$

Note the general formula as for one dimension:

$$
e^{t L(D)} e^{z \cdot x}=e^{t L(z)} e^{z \cdot x} .
$$

By the above remarks,

$$
e^{z \cdot C} 1=e^{t L} e^{z \cdot x} e^{-t L} 1=e^{t L} e^{z \cdot x}=e^{t L(z)} e^{z \cdot x}
$$

as required.

ExAMPLES. Here $\left(x_{1}, x_{2}\right)=(x, y),\left(z_{1}, z_{2}\right)=(z, w)$.

1. $L=D_{x} D_{y} . \quad \Lambda_{1}=z D_{y} . \quad \Lambda_{2}=w D_{x}$

and

$$
\begin{aligned}
e^{z \cdot C} f & =e^{w y} e^{t w D_{x}} e^{z x} f(x, y+z t) \\
& =e^{w y+z x} e^{z t w} f(x+w t, y+z t) .
\end{aligned}
$$

2. $L=D_{x}-(1 / 2) D_{y}^{2} . \quad \Lambda_{1}=z . \quad \Lambda_{2}=-\left(w^{2} / 2\right)-w D_{y}$

and

$$
\begin{aligned}
e^{z \cdot C} f & =e^{z x+w y} e^{z t-w^{2} t / 2} f(x, y-w t) \\
& =e^{z(x+t)} e^{w y-w^{2} t / 2} f(x, y-w t) .
\end{aligned}
$$

VIII. Further examples and remarks.

A. Homogeneity.

If the generator $L$ has homogeneity such that $a L(D)=L\left(a^{c} D\right)$, then

$$
e^{z \cdot c} 1=e^{z \cdot x+t L(z)}
$$

becomes, substituting $z \rightarrow a^{c} z$,

$$
e^{a^{c} z \cdot x+t L\left(a^{c} c_{z}\right.}=e^{z \cdot a^{c} x+a t L(z)} .
$$

Thus,

$$
h_{k}\left(a^{c} x, a t\right)=a^{k c} h_{k}(x, t)
$$

or equivalently $t^{k c} h_{l k}(x, 1)=h_{k}\left(x t^{c}, t\right)$, for every $k$.

On $R^{N}, a L(D)=L\left(a^{c} D\right)$ means 


$$
L\left(a^{c_{1}} D_{1}, \cdots, a^{c_{N}} D_{N}\right)=a L\left(D_{1}, \cdots, D_{N}\right),
$$

and $a^{k c}=a^{k \cdot c}$.

B. Higher Order Operators. Extensions.

Consider the equation

$$
\frac{\partial u}{\partial z}=x u+\frac{t}{2} u^{\prime \prime}
$$

This is of the type

$$
\frac{\partial u}{\partial z}=C u \text { with } L=\frac{D^{3}}{6} .
$$

So the solution is

$$
u=e^{z C} f=e^{z x} e^{t} f=e^{z x+z^{3} t / 6} \int f\left(x+\frac{z^{2} t}{2}+y \sqrt{z t}\right) \frac{e^{-y^{2 / 2}}}{\sqrt{2 \pi}} d y .
$$

On the other hand, for convenience setting $t=1$, the Feynman-Kac formula gives for Brownian motion $b(z)$,

$$
u=\left\langle f(x+b(z)) e^{\int_{0}^{z}(x+b(s) d s}\right\rangle .
$$

This suggests considering operators $C$ of the form $\psi(x)+t \dot{\phi}(D)$. In case either $\psi$ or $\phi$ generates a process, then the solution is of (generalized) Feynman-Kac type. By Fourier duality,

$$
\text { for } C=\psi(x)+t \dot{\phi}(D), \quad \text { set } \widehat{C}=\psi\left(i \frac{d}{d \xi}\right)+t \dot{\phi}(i \xi) \text {. }
$$

Denote by $p$ the process generated by $\phi(D)$ and by $q$ the process generated by $\psi(i(d / d \xi))$-assuming that these are well-defined in some sense.

Set $u=e^{z C} f$ and $v=\widehat{u}=e^{z \ell} \hat{f}$.

$$
\frac{\partial u}{\partial z}=\psi(x) u+t \dot{\phi}(D) u \text { and } \frac{\partial v}{\partial z}=\psi\left(i \frac{d}{d \xi}\right) v+t \phi(i \xi) v .
$$

Then with $\langle>$ corresponding to expectation with respect to the process indicated,

$$
\begin{aligned}
& u=\left\langle f(x+p(z t)) e^{\int_{0}^{z} \psi(x+p(s t)) d s}\right\rangle \\
& v=\left\langle\hat{f}(\xi+q(z)) e^{t} \int_{0}^{z} \int^{(i \xi+i q(s)) d s}\right\rangle .
\end{aligned}
$$

Applying Fourier transformation to $v$ and $u$ yields the dual representations: 


$$
\begin{aligned}
& u=\left\langle e^{-i q(z) x} e^{\left.t \int_{0}^{z} \phi(D+i(q(s))-q(z))\right) d s}\right\rangle f \\
& v=\left\langle e^{i \xi p(z t)} e^{\int_{0}^{z} \psi\left(i D_{\xi}+p(s t)-p(z t)\right) d s}\right\rangle \hat{f} .
\end{aligned}
$$

The solution $u$ is represented in terms of either the $p$ or $q$ process.

In particular, then, if processes are defined for generators of, say, polynomial type of degree $<n$, then any process generated by a polynomial of degree $n$ should satisfy the constraints imposed by the above duality requirements. E.g. For deg $\phi=n$, define $p$ implicitly by requiring $u(p)=u(q)$, using $\phi$ as a potential for all choices of $\psi, \operatorname{deg} \psi<n$.

In this way, inductively, a process and corresponding stochastic calculus is built up for all polynomial generators $L(D)$. By appropriate limiting procedures, extensions to operators with continuous generators and variable coefficients would result.

For example, for $\phi=D^{3} / 6, \psi=-x^{2} / 2, b(z)$ Brownian motion,

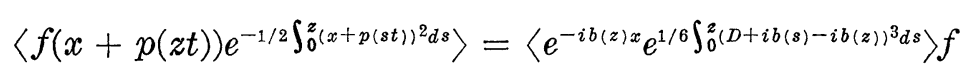

for all suitable functions $f$.

C. Relation with field theory.

In case $L$ is skew-adjoint, $C$ will be self-adjoint and conversely any set-up with Hermitian operators such that $[D, C]=1$ is essentially of the above type by Stone-Von Neumann. $C$ is the "creation operator" and $D$ the "annihilation operator", $A$ the "number operator"; $h_{n}$ are the Wick products. See, e.g., Simon [7] and references there, Segal [5, 6] and Miller [3].

IX. Functional calculus for C. Fundamental operators $\eta_{p}$. From $\S \mathrm{V}$,

$$
e^{z C}=e^{t L} e^{z x} e^{-t L} .
$$

Expanding in powers of $z$ yields

$$
C^{n}=e^{t L} x^{n} e^{-t L}
$$

In particular

$$
C=e^{t L} x e^{-t L} .
$$

So $\phi(C)$ is defined for any function $\phi$ for which

$$
\dot{\phi}(C)=e^{t L} \phi(x) e^{-t L} \text { makes sense. }
$$

Note that in particular, $\phi(C) f$ can be defined for harmonic $f$ by

$$
\dot{\phi}(C) f=e^{t L} \dot{\phi}(x) f .
$$


Defining $C$ directly, as above, yields an operator for any $L$ that generates a group; for general Markov semigroups, with possibly variable-coefficient generators, $\phi(C) f$ is defined for harmonic $f$ at least.

Before defining the fundamental operators, first recall Leibniz' rule for differentiating a product. It yields the following:

Lemma. (General Leibniz formula.)

As operators,

$$
\psi(D) \phi(x)=\sum_{p \geqq 0} \frac{1}{p !} \frac{\partial^{p} \phi}{\partial x^{p}} \frac{\partial^{p} \psi}{\partial D^{p}}
$$

Consequently,

$$
[\psi(D), \phi(x)]=\sum_{p \geqq 1} \frac{1}{p !} \frac{\partial^{p} \dot{\phi}}{\partial x^{p}} \frac{\partial^{p} \psi}{\partial D^{p}}
$$

Note. Assumptions on $\psi$ are $\phi$ are not stated explicitly, depending on the domains involved. For the proof, it is assumed that is can be approximated by polynomials in some reasonable manner. See, e.g., recent notes on pseudo-differential operators, Taylor [10]also references are there to papers by Kohn, Nirenberg, and Hormander-for technical clarifications concerning the generalized Leibniz formula.

Proof.

$$
D^{r} \phi=\Sigma\left[\begin{array}{l}
r \\
k
\end{array}\right] \frac{\partial^{k} \phi}{\partial x^{k}} D^{r-k} \text { by basic Leibniz' rule } .
$$

Or,

$$
D^{r} \phi=\sum \frac{1}{k !} \frac{\partial^{k} \phi}{\partial x^{k}} \frac{\partial^{k} D^{r}}{\partial D^{k}}
$$

For $\psi \sim \sum c_{r} D^{r}$

$$
\psi^{\prime} \dot{\phi}=\sum \sum \frac{1}{k !} \frac{\partial^{k} \phi}{\partial x^{k}} \frac{\partial^{k}}{\partial D^{k}} c_{r} D^{r}=\sum \frac{1}{k !} \frac{\partial^{k} \phi}{\partial x^{k}} \frac{\partial^{k} \psi}{\partial D^{k}}
$$

Using this formula,

$C=e^{t L} x e^{-t L}=x+\left[e^{t L}, x\right] e^{-t L}=x+t L^{\prime}$ as previously defined.

And

$$
\phi(C)=e^{t L} \phi(x) e^{-t L}=\sum_{p \geqq 0} \frac{1}{p !} \frac{\partial^{p} \phi}{\partial x^{p}} \eta_{p}(D, t)
$$


defining

$$
\eta_{p}(D, t)=e^{-t L} \frac{\partial^{p}}{\partial D^{p}} e^{t L}
$$

These are the fundamental operators.

In particular,

$$
C^{n}=\Sigma\left[\begin{array}{l}
n \\
p
\end{array}\right] x^{n-p} \eta_{p}
$$

It is now seen that the moments

$$
\mu_{r}=\int y^{r} p_{t}(y)=\eta_{r} 1
$$

This enables the formulation of the basic theorem as follows.

HARMONICITY THEOREM.

The following are equivalent:

(1) $f$ is harmonic.

(2) $e^{z C} f$ is harmonic.

(3) $\phi(C) f$ is harmonic for all suitable $\phi$.

(4) $\sum\left[\begin{array}{l}n \\ p\end{array}\right] x^{n-p} \eta_{p} f$ is harmonic for $n \geqq 0$.

Proof.

$1 \Rightarrow 2$ and 3 :

For $u=\phi(C) f$, $\frac{\partial u}{\partial t}=L u+e^{t L} \phi(x) e^{-t L}\left(\frac{\partial}{\partial t}-L\right) f=L u$, as for Remark $\# 2$ in $\S \mathrm{V}$.

$3 \Rightarrow 4:$ follows from the above formula for $C^{n}$ via the general Leibniz lemma.

$4 \Rightarrow 1: \quad \eta_{0}=$ identity.

EXAMPLE. The gamma distributions. Consider the case

$$
\begin{gathered}
P_{t}(y)=\frac{y^{t-1} e^{-y}}{\Gamma(t)}(y \geqq 0) \\
\int e^{i \xi y} P_{t}(y)=\left[\frac{1}{1-i \xi}\right]^{t} .
\end{gathered}
$$

So $L=-\log (1-D), L^{\prime}=(1-D)^{-1}$. And

$$
\begin{aligned}
\eta_{p}= & (1-D)^{t} \frac{\partial^{p}}{\partial D^{p}}(1-D)^{-t}=\frac{(-1)^{p}(-t) !}{(-t-p) !}(1-D)^{-p} \\
& =t(t+1) \cdots(t+p-1)(1-D)^{-p} .
\end{aligned}
$$




$$
C^{n}=(1-D)^{-t} x^{n}(1-D)^{t}
$$

and

$$
C^{n} 1=(1-D)^{-t} x^{n} .
$$

Now it is easy to see that setting $t=-n$ yields $q_{n}(x)=$ $\left(x-n L^{\prime}\right)^{n} 1=(1-D)^{n} x^{n}$ an orthogonal system on $[0, \infty)$ relative to $e^{-x}$, i.e., they are essentially the Laguerre polynomials. Recall the definition of $C^{n} 1$ in terms of moments of $P_{t}$. This yields formally

$$
q_{n}(x)=\int_{0}^{\infty}(x+y)^{n} \frac{y^{-n-1} e^{-y}}{\Gamma(-n)} d y
$$

with

$$
\mu_{r}=\frac{1}{\Gamma(-n)} \int_{0}^{\infty} y^{r-n-1} e^{-y} d y .
$$

From the formula for $\eta_{n}$,

$$
\mu_{r}=\eta_{r} 1=\frac{(-1)^{r} n !}{(n-r) !}(1-D)^{-r} 1=\frac{(-1)^{r} n !}{(n-r) !}
$$

since $(1-D)^{-1} 1=1$.

If the gamma function were defined for negative integers, the first formula for $\mu_{r}$ would give

$$
\mu_{r}=\frac{\Gamma(r-n)}{\Gamma(-n)}=\frac{(-1)^{r} n !}{(n-r) !} .
$$

This shows that it would be consistent to define

$$
\Gamma(-n)=\frac{(-1)^{n}}{n !} \text { if only ratios are computed. }
$$

That is, define $\Gamma(0)=\zeta$, with $0 \cdot \zeta=0 \Gamma(0)=\Gamma(1)=1$. Then setting

$$
\Gamma(-n)=\frac{(-1)^{n}}{n !} \zeta
$$

also preserves the basic property $x \Gamma(x)=\Gamma(x+1)$ as indicated by the computation of $\mu_{r}$. Note that $\Gamma$ has simple poles at $-n$ for $n \geqq 0$ with residue $(-1)^{n} / n !$.

Finally, note the difference between the Laguerre and Hermite systems.

$H_{n}(x, t)=(x-t D)^{n} 1$ are orthogonal for $t>0$ for the measure with density $e^{-x^{2} / 2 t}$ while $L_{n}(x)=\left(x-n(1-D)^{-1}\right)^{n} 1$ requires setting $t=-n$ to have an orthogonal system with respect to the single density $e^{-x}(x \geqq 0)$. 
Generally, $H_{n}=\left(x-t L^{\prime}\right)^{n} 1$ does satisfy a generalized three-term recurrence relation,

$$
x H_{n}=t L^{\prime} H_{n}+H_{n+1} .
$$

IXa. Appendix to Section IX. Computing $\eta_{p}$. The following formula for computing the $\eta$ 's is convenient in the case $L$ is analytic.

Proposition.

$$
\eta_{N}=\sum_{\substack{\Sigma k p_{k}=N \\
p_{k} \geq 0}} \frac{t^{p_{1}+\cdots+p_{k}}}{\prod_{k}(k !)^{p_{k}}}\left[\begin{array}{c}
N \\
p_{1} \cdots p_{N}
\end{array}\right] L_{1}^{p_{1}} L_{2}^{p_{2}} \cdots L_{N}^{p_{N}}
$$

where

$$
L_{j}=\left[\frac{\partial}{\partial D}\right]^{j} L
$$

Proof. $\eta_{N}=e^{-t L}[\partial / \partial D]^{N} e^{t L}$. Multiply by $a^{N} / N$ ! in the formula above and sum. The left-hand side becomes:

$$
e^{t(L(D+a)-L(D))}=\exp \left[t \sum_{N=1}^{\infty} \frac{a^{N} L_{N}}{N !}\right] .
$$

Expanding the exponential and collecting terms corresponding to each $a^{N}$ yields the formula.

REMARK. Observe that, for $t=1$, the coefficient of $L_{1}^{p_{1}} \cdots L_{N}^{p_{N}}$ is the number of ways of grouping $N$ elements to have $p_{k}$ groups of $k$ elements each.

$X$. The number operator. Vacuum functions. This section continues $\S \mathrm{VI}$ as a further study of the operator $A$. Recall that in one dimension

$$
A=C D=e^{t L} x e^{-t L} D=e^{t L} x D e^{-t L} .
$$

In the multi-dimensional case, the vector operator $A$ acts in each coordinate. As seen in $\S \mathrm{VI}, A f=0$ implies that $A_{j} f_{k}=k_{j} f_{k}$ for each multi-index $k$. A scalar operator can be defined that acts similarly.

DEFINITION. The number operator is the scalar operator defined by

$$
A=C \cdot D=\sum C_{j} D_{j}=\sum A_{j} .
$$

In the following, $A$ will denote this scalar operator; $A_{j}$ will 
denote components of the original vector operator.

Proposition.

(1) $A_{j} f=0$ for $1 \leqq j \leqq N$ implies $A f_{k}=|k| f_{k}$.

(2) In fact, $A f=0$ implies $A f_{k}=|k| f_{k}$.

Proof.

(1) follows from above remarks.

(2) $A e^{z \cdot C} f=e^{t L} x \cdot D e^{z \cdot x} e^{-t L} f$

$$
\begin{aligned}
& =e^{t L} x \cdot z e^{z \cdot x} e^{-t L} f+e^{t L} e^{z \cdot x} x \cdot D e^{-t L} f \\
& =z \cdot D_{z} e^{t L} e^{z \cdot x} e^{-t L} f .
\end{aligned}
$$

Since,

$$
e^{t L} e^{z \cdot x} x \cdot D e^{-t L} f=e^{z \cdot c} A f=0 .
$$

The conclusion follows as in the one-dimensional case.

DEFINITION. A vacuum function $f(t, x)$ satisfies by definition:

(1) $\partial f / \partial t=L f$, i.e., $f$ is harmonic.

(2) $A f=0$.

Thus, $f_{k}$ are harmonic functions and eigenfunctions of $A$ as well. The usual choice for the vacuum, as seen previously, is the constant function 1. However, in singular cases, e.g., Example 4 of $\S I V$, other choices may be necessary.

In the case $f$ is independent of $t$, the vacuum conditions simplify.

Proposition. When $\partial f / \partial t=0, f$ is a vacuum function if and only if

(1) $L f=0$.

(2) $x \cdot D f=0$.

Proof. To verify (2). Note that since $f$ is independent of $t$, setting $t=0$ in the equation $A f=0$ reduces to (2).

Conversely, assuming (1) and (2) yields:

$$
A f=e^{t L} x \cdot D e^{-t L} f=e^{t L} x \cdot D f=0 .
$$

Regarding equation (2) of the above proposition, note the following.

Proposition. $x \cdot D f=0$ if and only if $f$ is homogeneous of degree zero, i.e.,

$$
f(\lambda x)=f(x) \text { for scalar } \lambda .
$$

Proof. $x \cdot D f=0$ implies $e^{\alpha x \cdot D} f=f(x)=f\left(e^{\alpha} x\right)$, for scalar $\alpha$.

Differentiating $f(\lambda x)=f(x)$ with respect to $\lambda$ yields the converse. 
A "general solution" is thus of the form

$$
f(x)=\sum_{a \in \mathbf{R}^{N}} Q_{a}\left(x_{1}^{a_{1}} x_{2}^{a_{2}} \cdots x_{N}^{a}\right)
$$

with $a$ 's such that $\sum a_{j}=0$ and the $Q_{a}$ functions of one variable. Note that any analytic $f$ homogeneous of degree zero must be constant.

\section{EXAmples.}

1. Brownian notion. $L=1 / 2 \sum D_{j}^{2} . \quad C_{j}=x_{j}+t D_{j}$. $A=C \cdot D=\sum\left(x_{j} D_{j}+t D_{j}^{2}\right)$. Since any harmonic $f$ is analytic, the only vacuums are constant functions.

$$
\text { 2. } L=D_{x} D_{y} . \quad C_{x}=x+t D_{y} . \quad C_{y}=y+t D_{x} .
$$

$A=x D_{x}+2 t D_{x} D_{y}+y D_{y}$. A harmonic function is of the form

$$
f=F(x)+G(y) \text {. }
$$

And $x \cdot D f=0$ yields

$$
x F^{\prime}(x)+y G^{\prime}(y)=0
$$

or

$$
x F^{\prime \prime}(x)=\text { constant }=-y G^{\prime}(y) .
$$

Choosing the particular solutions

$$
F=\log x, \quad G=-\log y
$$

yields

$$
f=\log \frac{x}{y}
$$

So

$$
u=e^{z C_{x}+w C_{y}} f=e^{w y+z x+t w z} \log \frac{x+w t}{y+z t}
$$

is harmonic and $A u=\left(z D_{z}+w D_{w}\right) u$.

$$
\text { For } h_{n m}=C_{x}^{n} C_{y}^{m} f, A h_{n m}=(n+m) h_{n m} \text {. }
$$

E.g.,

$$
\begin{aligned}
& h_{10}=x(\log x-\log y)-t / y, \quad A h_{10}=h_{10} . \\
& h_{11}=(x y+t)(\log x-\log y), \quad A h_{11}=2 h_{11} .
\end{aligned}
$$

3. $L=D_{x}-D_{y}^{2} . \quad C_{x}=x+t . \quad C_{y}=y-2 t D_{y}$.

$$
A=x D_{x}+t D_{x}+y D_{y}-2 t D_{y}^{2} \text {. }
$$

For $f$, homogeneous of degree zero, of the form

$$
f=Q\left[\frac{x}{y}\right]
$$


to be harmonic yields the equation

$$
y Q^{\prime}+2\left[\frac{x}{y}\right] Q^{\prime}+\left[\frac{x}{y}\right]^{2} Q^{\prime \prime}=0 .
$$

Thus, $y Q^{\prime}=0$ and $Q$ must be constant.

In case $f$ depends on $t$, the remark in $\S \mathrm{V}$ regarding the 2 nd series indicates the canonical choice

$$
f=D^{-1} p_{t}(-x)=\int_{-\infty}^{x} p_{t}(-y) d y=\int_{-\infty}^{\infty} p_{t}(y) d y
$$

Harmonicity follows immediately from

$$
p_{t}(-x)=\frac{1}{2 \pi} \int e^{i \xi x} e^{t L(i \xi)}
$$

and the commutativity of the operators $\partial / \partial t, L$, and $D^{-1}$.

\section{REFERENCES}

1. E. McBride, Obtaining Generating Functions, Springer-Verlag, New York, 1971.

2. H. P. McKean, Geometry of differential space, Annals of Prob., 1, \#2, (1973), 197-206.

3. Willard Miller, On the special function theory of occupation number space, Comm. Pure \& Appl. Math., XVIII (1965), 679-696.

4. Ray Redheffer, Induced transformations of the derivative-vector, Amer. Math. Monthly, 83 \#4, 255-259.

5. I. Segal, Non-linear functions of weak processes, I. J. Functional Analysis, 4 (1969), 404-451.

6. Non-linear functions of weak processes, II, J. Functional Analysis, 6 (1970), 29-75.

7. Barry Simon, The $P(\phi)_{2}$ Euclidean (Quantum) Field Theory, Princeton Univ. Press, 1974.

8. D. Stroock and S. R. S. Varadhan, Diffusion processes with continuous coefficients I, Comm. Pure \& Appl. Math., XII (1969), 345-400.

9. - Diffusion processes with continuous coefficients II, Comm. Pure \& Appl. Math., XII (1969), 479-530.

10. Michael Taylor, Pseudo-Differential Operators, Springer-Verlag, Lecture Notes in Mathematics, \#416.

11. S. R. S. Varadhan, Stochastic Processes, CIMS Lecture Notes, New York University, 1968.

12. D. V. Widder, The role of the Appell transformation in the theory of heat conduction, Trans. Amer. Math. Soc., 109 (1963), 121-134.

13. S. J. Wolfe, On Moments of Probability Distribution Functions, in Fractional Calculus and Its Applications, Springer-Verlag Lecture Notes in Mathematics \#457, 306-316.

Received November 16, 1976.

UNIVERSITY OF UTAH

Salt Lake City, UT 84112 


\section{PACIFIC JOURNAL OF MATHEMATICS}

\section{EDITORS}

RICHARD ARENS (Managing Editor)

University of California

Los Angeles, California 90024

C. W. CurTis

University of Oregon

Eugene, OR 97403

C. C. MOORE

University of California

Berkeley, CA 94720
J. DUGUNDJI

Department of Mathematics University of Southern California Los Angeles, California 90007

R. FinN AND J. Milgram Stanford University Stanford, California 94305

\section{ASSOCIATE EDITORS}

E. F. BECKENBACH

B. H. NeumanN

F. WOLF

K. YoSHIDA

\section{SUPPORTING INSTITUTIONS}

UNIVERSITY OF BRITISH COLUMBIA CALIFORNIA INSTITUTE OF TECHNOLOGY UNIVERSITY OF CALIFORNIA MONTANA STATE UNIVERSITY UNIVERSITY OF NEVADA, RENO NEW MEXICO STATE UNIVERSITY OREGON STATE UNIVERSITY UNIVERSITY OF OREGON
UNIVERSITY OF SOUTHERN CALIFORNIA

STANFORD UNIVERSITY

UNIVERSITY OF HAWAII

UNIVERSITY OF TOKYO

UNIVERSITY OF UTAH

WASHINGTON STATE UNIVERSITY

UNIVERSITY OF WASHINGTON 


\section{Pacific Journal of Mathematics \\ Vol. 78, No. $1 \quad$ March, 1978}

Simeon M. Berman, A class of isotropic distributions in $\mathbf{R}^{n}$ and their

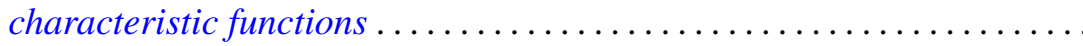

Ezra Brown and Charles John Parry, The 2-class group of biquadratic fields.

II ........................................ 11

Thomas E. Cecil and Patrick J. Ryan, Focal sets of submanifolds ....... 27

Joseph A. Cima and James Warren Roberts, Denting points in $B^{p} \ldots \ldots \ldots 41$

Thomas W. Cusick, Integer multiples of periodic continued fractions . . . . . 47

Robert D. Davis, The factors of the ramification sequence of a class of

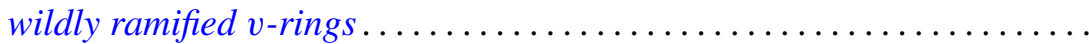

Robert Martin Ephraim, Multiplicative linear functionals of Stein

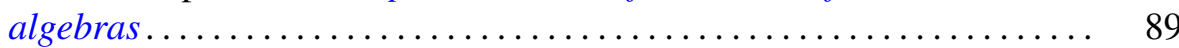

Philip Joel Feinsilver, Operator calculus . .................... 95

David Andrew Gay and William Yslas Vélez, On the degree of the splitting field of an irreducible binomial ..........................

Robert William Gilmer, Jr. and William James Heinzer, On the divisors of

monic polynomials over a commutative ring ..................

Robert E. Hartwig, Schur's theorem and the Drazin inverse .............

Hugh M. Hilden, Embeddings and branched covering spaces for three and four dimensional manifolds ............................

Carlos Moreno, The Petersson inner product and the residue of an Euler product. ...

Christopher Lloyd Morgan, On relations for representations of finite groups....

Ira J. Papick, Finite type extensions and coherence

$\mathrm{R}$. Michael Range, The Carathéodory metric and holomorphic maps on a class of weakly pseudoconvex domains ................

Donald Michael Redmond, Mean value theorems for a class of Dirichlet series

Daniel Reich, Partitioning integers using a finitely generated semigroup ...

Georg Johann Rieger, Remark on a paper of Stux concerning squarefree

numbers in non-linear sequences

Gerhard Rosenberger, Alternierende Produkte in freien Gruppen ..

Ryōtarō Satō, Contraction semigroups in Lebesgue space

Tord Sjödin, Capacities of compact sets in linear subspaces of $\mathbf{R}^{n}$

Robert Jeffrey Zimmer, Uniform subgroups and ergodic actions of exponential Lie groups......................... 